\title{
Exact Analysis of Single-Wavelength Optical Buffers With Feedback Markov Fluid Queues
}

\author{
Huseyin Emre Kankaya and Nail Akar
}

\begin{abstract}
Optical buffering via fiber delay lines is used for contention resolution in optical packet and optical burst switching nodes. This article addresses the problem of exactly finding the blocking probabilities in an asynchronous single-wavelength optical buffer. Packet lengths are assumed to be variable and modeled by phase-type distributions, whereas the packet arrival process is modeled by a Markovian arrival process that can capture autocorrelations in interarrival times. The exact solution is based on the theory of feedback fluid queues for which we propose numerically efficient and stable algorithms. We not only find the packet blocking probabilities but also the entire distribution of the unfinished work in this system from which all performance measures of interest can be derived.
\end{abstract}

Index Terms-Fiber delay line (FDL); Buffer; Optical packet switching; Optical burst switching; Feedback fluid queues.

\section{INTRODUCTION}

$\mathbf{O}$ ptical packet switching-based paradigms are beginning to mature as they attempt to more efficiently utilize the fiber capacity as opposed to their optical circuit switching counterparts. Two such paradigms have recently been proposed: optical packet switching (OPS) [1] and optical burst switching (OBS) [2]. In this article, we analytically study the performance of an optical packet (or burst) switch that uses fiber delay lines.

OPS/OBS can either be synchronous (time slotted)

Manuscript received June 10, 2009; revised September 15, 2009; accepted September 19, 2009; published October 15, 2009 (Doc. ID 112523).

This work was carried out while H. E. Kankaya (e-mail: emre@ee.bilkent.edu.tr) was a Ph.D. student in the Electrical and Electronics Engineering Department, Bilkent University, Ankara, Turkey. H. E. Kankaya is now with Zirve University, Gaziantep, Turkey.

N. Akar (e-mail: akar@ee.bilkent.edu.tr) is with the Electrical and Electronics Engineering Department, Bilkent University, Ankara, Turkey.

Digital Object Identifier 10.1364/JOCN.1.000530 or asynchronous (unslotted). Optical packets are of fixed length in synchronous systems leading to a need for costly synchronization equipment. Synchronous switching is known to be efficient in terms of blocking performance due to the alignment of packet arrivals. On the other hand, optical packets are of variable length in asynchronous systems, and therefore packet arrivals need not be aligned. Asynchronous OPS appears to be a better fit as a transport technology for the next-generation Internet due to the variable lengths of IP packets in the Internet. In this paper, we focus on asynchronous OPS.

In OPS networks, contention arises when two or more incoming optical packets contend for the same output wavelength. Methods for contention resolution include wavelength conversion [3], deflection routing [2], or optical buffering [4], which is the scope of the current article. In electronic buffering, contending packets are stored in RAMs (random access memory) to be transmitted at a later time. However, optical RAMs are not feasible today. Instead, a well-known technique is to use fiber delay lines (FDLs) in which a contending packet is sent over a coil of fiber that gives the packet a fixed delay that is enough to resolve the contention. This paradigm is called optical FDL buffering, or simply optical buffering.

There are different architectures proposed for optical buffers based on their position in the switch, namely, input buffering, output buffering, and shared buffering [5]. Input buffering is known to have relatively lower performance than the other two. In output buffering, a set of FDLs is dedicated for each output port (or fiber). On the other hand, in shared buffering, FDLs can be shared by all output ports for improved performance. We focus on output buffering in this article. In particular, we focus on the case where a set of FDLs is dedicated for each wavelength (link) of an output port. This situation arises when

- each output port has a single wavelength,

- we have multiple wavelengths per output port but we do not use wavelength converters that would allow buffer sharing across different wavelengths. 
Hybrid use of wavelength converters and FDL sharing among multiple wavelengths of the same output port are left for future research. Optical buffers can be configured as degenerate or nondegenerate. In degenerate buffering, the lengths of FDLs are consecutive multiples of a parameter $D$, which is called the granularity parameter. The buffer size is denoted by $K$ and the $i$ th FDL, $i=1,2, \ldots, K$, introduces a delay of $i D$ to an incoming optical packet finding the output wavelength occupied. In nondegenerate buffering, the lengths of individual FDLs can be arbitrary and the $i$ th FDL exerts a delay of $D_{i}, i=1,2, \ldots, K$, where we have $D_{i}>D_{j}$ for $i>j$. Although we present an analytical method for the case of degenerate buffering only, the method we propose can directly be applied to the nondegenerate case.

We now present an example to demonstrate how this optical buffer works. For this purpose, we define $H(t)$ as the unfinished work (or channel horizon) at time $t$ and with all the admitted packets into the buffer at time $t$, we need to wait for at least $H(t)$ for the link to become idle. Let us now assume an optical packet that arrives at time $t$. If the link is idle at this arrival epoch, then $H(t)=0$ and the packet can immediately be forwarded over the link making $H\left(t^{+}\right)=B$, where $B$ is the length of the packet (in time units). If $H(t)>K D$, then the delays introduced by each delay line are not enough to resolve the contention and the packet will be dropped without a need to modify $H(t)$. The more interesting scenario is when $0<H(t) \leqslant K D$ in which case the arriving packet of length $B$ will be directed to the FDL that delays it by

$$
D\left\lceil\frac{H(t)}{D}\right\rceil,
$$

where $\lceil x\rceil$ is the smallest integer greater than or equal to $x$. Obviously, in this case, the unfinished work is modified as

$$
H\left(t^{+}\right)=D\left\lceil\frac{H(t)}{D}\right\rceil+B .
$$

At other times, the unfinished work $H(t)$ decreases at a unity rate until it hits zero and then stays at this level; i.e., unfinished work cannot be negative.

To illustrate the operation of a single-wavelength degenerate optical buffer, we depict a sample path of the channel horizon process in Fig. 1 for an example when $D=K=2$. Packet arrivals occur at time epochs $t$ $=1,3,4,5$, and 7 , with corresponding sizes of $3,2,2,2$, and 1 , respectively. The packet arriving at $t=5$ is blocked since $H(5)=5>K D=4$. All of the four other packets are accepted into the buffer. Note that an ac-

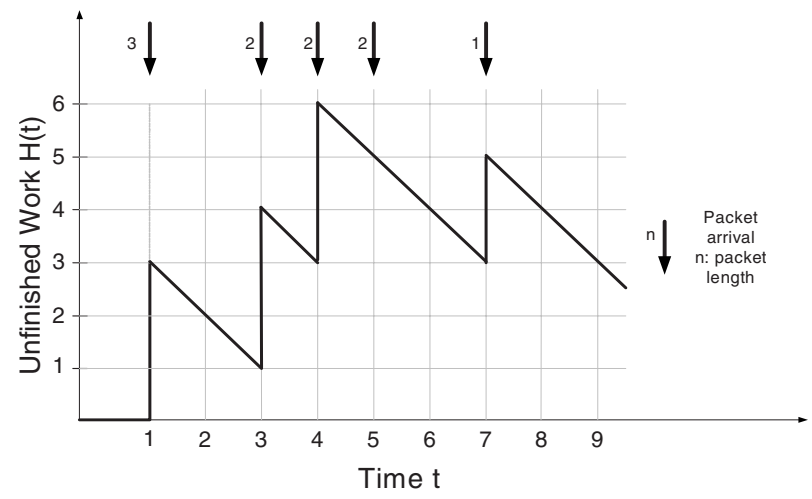

Fig. 1. Evolution of the channel horizon $H(t)$ for a singlewavelength degenerate optical buffer with $D=K=2$.

cepted optical packet leads to an immediate jump in $H(t)$, whereas a blocked packet does not have any effect.

Various analytical methods have been proposed to analytically evaluate the performance of an asynchronous single-wavelength optical buffer that differ from each other on the basis of (i) the stochastic model used for packet arrivals and packet lengths, (ii) whether $K$ is finite or infinite, and (iii) whether the analytical method is exact or approximate. One of the earlier works in [6] studies an optical buffer with general $K$ and with Poisson packet arrivals and exponential packet lengths and presents an approximate method using an iterative procedure that is simple to implement. For the same buffer, the work in [7] assumes generic distributions for packet interarrivals and services and the authors present a Markov chain model for solving the optical buffer. The entries of the Markov chain can be found by calculating certain definite integrals for which computational procedures are not elaborated. Moreover, depending on the distributions used in the model, the number of the states of the Markov chain can be excessive, which then calls for an approximate state reduction method for computational feasibility. In two special cases, the authors [7] have shown that their solution is exact: (i) exponential interarrivals and generic packet lengths and (ii) generic interarrivals and exponential packet lengths. Again, following similar traffic assumptions, the case of $K=\infty$ is studied by [8] to develop heuristics for the case of $K<\infty$ through formulas involving infinite sums. Three special cases corresponding to (i) exponentially distributed, (ii) deterministic, and (iii) mixtures of deterministic burst lengths are given for which the resulting formula does not involve an infinite sum and therefore the method turns out to be computationally effective. The work in [9] studies a finite capacity optical buffer in the special case of Poisson arrivals and generic packet lengths using Markov chains as in [7] but also provides closed-form expressions for blocking probability and expected delays, 
which is very valuable in terms of engineering insight. Note, however, that a finite-state Markov chain solution is available in the literature for only a number of special cases (see [7,9]), and there still is a need for exploring exact algorithms for analyzing optical buffers with more general traffic inputs. An approximate method based on the assumption $K=\infty$ is proposed in [10] based on the generating function approach for the case of Poisson arrivals and generally distributed packet lengths. The reference [11] studies an optical buffer with general packet size distributions where the method does not seem to work for all possible load values and the traffic is limited to Poisson arrivals only.

In this article, we exactly find the steady-state distribution of the unfinished work in asynchronous single-wavelength optical buffers from which all steady-state performance measures such as packet blocking probability, expected delay, etc., can be calculated. The stochastic tool that we use is the wellknown multiregime Markov fluid queues [12,13], and to the best of our knowledge this theory has not been applied to optical buffers before. Multiregime Markov fluid queues are more complex and are less explored than Markov chains, but the model we propose is a finite state-space multiregime Markov fluid queue for optical buffers with more general traffic inputs than those studied in the literature. On the other hand, in this model we need to keep track of the horizon parameter (or unfinished work) as opposed to actual waiting times that take values from a finite set, but keeping track of the continuous horizon parameter has helped us build a finite state-space Markov fluid queue model resulting in an exact matrix-analytical algorithm for solving optical buffers with very general traffic input. Our assumptions and contributions are listed below.

Packet interarrival times are modeled by the versatile Markovian arrival process (MAP) by which one can capture dependence between successive interarrivals $[14,15]$. The MAP generalizes the Poisson process by allowing nonexponential interarrival times while maintaining the underlying Markovian structure. To define a MAP, we first consider a Markov chain with infinitesimal generator $\widetilde{D}=D_{0}+D_{1}$ for which all off-diagonal entries of $D_{0}$ and all entries of $D_{1}$ are nonnegative. Transitions associated with $D_{1}$ are the epochs of arrivals in a MAP characterized with the pair $\left(D_{0}, D_{1}\right)$. For more details, we refer the reader to [14]. Let $\pi$ be the stationary probability vector of the phase process with generator $\widetilde{D}$ so that $\pi$ satisfies $\pi \widetilde{D}=0, \pi e=1$, where $e$ is a column vector of ones of appropriate size. The mean arrival rate $\lambda$ for a MAP is $\lambda=\pi D_{1} e$ [15]. The MAP arrival model is more general than the stochastic models used for the analysis of op- tical buffers, and it can especially be useful to demonstrate the impact of autocorrelations (in interarrival times) on system performance. Note that autocorrelations in packet interarrival times and their nonPoisson nature has been well-known for Internet traffic $[16,17]$.

The packet length $B$ is modeled by a phase type (PH-type) distribution [18]. Consider a Markov process on the states $\{1,2, \ldots, s, s+1\}$ with initial probability vector $[v, 0], v=\left[v_{1}, v_{2}, \ldots, v_{s}\right]$, and infinitesimal generator

$$
Q_{P H}=\left[\begin{array}{cc}
S & S^{0} \\
0 & 0
\end{array}\right],
$$

where $S$ is an $s \times s$ nonsingular matrix, $S^{0}$ is $s \times 1$, and $\mathrm{Se}+S^{0}=0$. The time till absorption into the absorbing state $s+1$ is a random variable $B$ that is said to have a $\mathrm{PH}$-type distribution with representation $(v, S)$ with order $s$ whose distribution function is written as $F_{B}(x)=1-v e^{S x} e, \quad x \geqslant 0$. PH-type distributions are known to be dense in the field of all positive-valued distributions and this feature of $\mathrm{PH}$-type distributions makes it a very suitable tool to model variable-size optical packets. Denseness of PH-type distributions means that given an arbitrary positive-valued distribution, one can approximate it with a PH-type distribution with any desired accuracy [14]. The reference [19] proposes an algorithmic procedure for obtaining $\mathrm{PH}$-type approximative distributions from empirical data and also other distributions. However, there is one notable deficiency of phase-type modeling; the approximate model may have large orders. For example, a deterministic distribution can be approximated by an Erlang- $k$ distribution with order $k$, but the order parameter $k$ may need to be very large if high accuracy is sought. Moreover, in next-generation optical networks, packet lengths can take values from a limited range or even they can take fixed values [9] for which $\mathrm{PH}$-type approximations may not be most effective for packet length modeling in the context of analysis of optical buffers. The scope of this work, however, is confined to PH-type distributed optical packet lengths and potential use of Markov fluid queues for more general packet lengths is left for future research.

Moreover,

- We do not make any assumptions about the number of FDLs $K$. In particular, we do not need the assumption $K=\infty$ as pursued in some earlier work. With this, we can also analyze situations where one only has a few FDLs.

- The method we use is based on the well-known theory of feedback fluid queues [12] and the numerically stable and efficient solution techniques we had proposed for such queues [13]. We intro- 
duce a number of modifications and enhancements to the method described in [13] that are necessary to solve the problem of interest in the current article.

- The method we propose is exact for MAP packet arrivals and PH-type distributed packet lengths.

Based on the algorithmic procedure we propose for optical buffers, we also obtain numerical results that help us provide provisioning guidelines for optical buffers.

The remainder of the article is organized as follows. Feedback fluid queues are described in Section II. In Section III, we provide the analytical model. Numerical results are presented in Section IV. We conclude in the final section.

\section{FEEDBACK MARKOV FLUID QueUES}

Markov fluid queues (MFQs) are described by a joint Markovian process $\{C(t), M(t) ; t \geqslant 0\}$, where $\{C(t)$; $t \geqslant 0\}$ refers to the queue occupancy and $\{M(t) ; t \geqslant 0\}$ is an underlying continuous-time Markov chain that determines the rate at which the buffer content $C(t)$ changes. The process $\{M(t) ; t \geqslant 0\}$ is called the background (or modulating) process of the MFQ. The reference [20] studies MFQs with infinite queue sizes using a spectral expansion approach, whereas [21] extends this analysis to finite queue sizes.

More general models, known as feedback Markov fluid queues (FMFQs), were introduced in [22,23], where both the rate of change of the buffer content and the background process are allowed to depend on the instantaneous queue occupancy. In this article, we concentrate on multiregime FMFQs, where the feedback has a piecewise-constant form [12]. The following is based on $[12,13]$. In multiregime FMFQs (we will use the term FMFQ in short to refer to a multiregime FMFQ in this paper), we have $K$ intermediate boundary points (or thresholds) and two terminal boundary points at the origin and infinity, respectively, i.e., 0 $=T^{(0)}<T^{(1)}<\cdots<T^{(K)}<T^{(K+1)}=\infty$. The queue is said to be in regime $k$ (at threshold $T^{(k)}$ ) if $T^{(k-1)}<C(t)<T^{(k)}$ $\left(C(t)=T^{(k)}\right)$. We assume that the modulating process $\{M(t) ; t \geqslant 0\}$ has a finite state space $\{1,2, \ldots, M\}$. When the system is in regime $k$ (at threshold $T^{(k)}$ ) then the background process $M(t)$ behaves according to a Markov process with generator $Q^{(k)}\left(\widetilde{Q}^{(k)}\right)$. The drift (net rate of change of the queue) while at state $m, 1 \leqslant m$ $\leqslant M$, in regime $k$ (at threshold $T^{(k)}$ ) is denoted by $r_{m}^{(k)}$ $\left.{ }^{\left({ }^{(k)}\right.}\right)$. We let $R^{(k)}\left(\widetilde{R}^{(k)}\right)$ be the diagonal matrix of drifts in regime $k$ (at threshold $T^{(k)}$ ). The dynamics of the buffer content for the FMFQ is then given by

$$
\frac{\mathrm{d} C(t)}{\mathrm{d} t}= \begin{cases}\max \left(0, \widetilde{r}_{M(t)}^{(0)}\right) & \text { if } C(t)=0, \\ r_{M(t)}^{(k)} & \text { if } T^{(k-1)}<C(t)<T^{(k)}, \\ \widetilde{r}_{M(t)}^{(k)} & \text { if } C(t)=T^{(k)} .\end{cases}
$$

We assume that the FMFQ has a unique steadystate solution, one of the necessary conditions of which dictates that the mean drift in regime $K+1$ should be negative. Let $f^{(k)}(y, t)$ denote the row vector of transient joint probability density functions (PDFs) at time $t$ in regime $k$ for $1 \leqslant k \leqslant K+1$, i.e., $f^{(k)}(y, t)$ $=\left[f_{1}^{(k)}(y, t), f_{2}^{(k)}(y, t), \ldots, f_{M}^{(k)}(y, t)\right]$, where

$$
\begin{gathered}
f_{m}^{(k)}(y, t)=\frac{\partial F_{m}^{(k)}(y, t)}{\partial y}, \quad T^{(k-1)}<y<T^{(k)}, \\
F_{m}^{(k)}(y, t)=P(C(t) \leqslant y, \quad M(t)=m), \quad T^{(k-1)}<y<T^{(k)},
\end{gathered}
$$

for $1 \leqslant k \leqslant K+1,1 \leqslant m \leqslant M$. The steady-state joint PDF can then be defined via taking the limit of Eq. (3) as $t \rightarrow \infty$, i.e., $f_{m}^{(k)}(y)=\lim _{t \rightarrow \infty} f_{m}^{(k)}(y, t)$. We then define the steady-state joint density vector

$$
f^{(k)}(y)=\left[f_{1}^{(k)}(y), f_{2}^{(k)}(y), \ldots, f_{M}^{(k)}(y)\right] .
$$

Similarly, we define $F_{m}^{(k)}(y)=\lim _{t \rightarrow \infty} F_{m}^{(k)}(y, t)$ and $F^{(k)}(y)=\left[F_{1}^{(k)}(y), F_{2}^{(k)}(y), \ldots, F_{M}^{(k)}(y)\right]$. Moreover, we define $c^{(k)}(t)$ to be the row vector of transient probability mass accumulations at the boundary point $T^{(k)}$ at time $t$ :

$$
c^{(k)}(t)=\left[c_{1}^{(k)}(t), c_{2}^{(k)}(t), \ldots, c_{M}^{(k)}(t)\right]
$$

where

$$
c_{m}^{(k)}(t)=P\left(C(t)=T^{(k)}, M(t)=m\right),
$$

for $0 \leqslant k \leqslant K, 1 \leqslant m \leqslant M$. The steady-state probability mass accumulations at the boundary points are defined by means of taking the limit of Eq. (7) as $t \rightarrow \infty$, i.e., $c_{m}^{(k)}=\lim _{t \rightarrow \infty} c_{m}^{(k)}(t), 0 \leqslant k \leqslant K, 1 \leqslant m \leqslant M$. We also define $c^{(k)}=\left[c_{1}^{(k)}, c_{2}^{(k)}, \ldots, c_{M}^{(k)}\right]$. Finally, we define the joint cumulative distribution function (CDF) $F_{m}(y)$ for all $y$ such that

$$
F_{m}(y)=F_{m}^{(k)}(y), \quad 1 \leqslant m \leqslant M, \quad \text { when } T^{(k-1)}<y<T^{(k)},
$$

and the queue occupancy $\mathrm{CDF}$ vector

$$
F(y)=\left[F_{1}(y), F_{2}(y), \ldots, F_{M}(y)\right] .
$$

Note that by definition, $F_{m}(y)$ and $F(y)$ are right continuous at the boundary points. The joint PDF $f_{m}(y)$ and the buffer occupancy PDF vector $f(y)$ are defined accordingly. The steady-state solution to the feedback fluid queue involves the calculation of $f^{(k)}(y)$ for $1 \leqslant k$ $\leqslant K+1$ and $c^{(k)}$ for $0 \leqslant k \leqslant K$. The feedback fluid queue of interest is illustrated in Fig. 2. 


\begin{tabular}{|c|c|c|c|}
\hline$\tilde{\mathbb{R}}^{(0)}, \tilde{Q}^{(0)}, c^{(0)}$ & & ${ }^{K-1)}, c^{(K-1)}$ & ${ }^{\prime}, c^{(K)}$ \\
\hline $\begin{array}{c}\text { Regime 1 } \\
R^{(1)}, Q^{(1)}, f^{(1)}(y)\end{array}$ & ..... & $\begin{array}{c}\text { Regime K } \\
R^{(K)}, Q^{(K)}, f^{(K)}(y)\end{array}$ & $\begin{array}{c}\text { Regime } \mathbf{K}+\mathbf{1} \\
R^{(K+1)}, Q^{(K+1)}, f^{(K+1)}(y)\end{array}$ \\
\hline
\end{tabular}

Fig. 2. The multiregime feedback fluid queue.

A spectral solution to the steady-state behavior, i.e., $f_{m}(\cdot), 1 \leqslant m \leqslant M$, of the FMFQ is given in [12]. This method requires the solution of $K+1$ eigenvalue problems for matrices of size $M$ and the solution of a matrix equation of size at most $(K+1) M$. In this method, all eigenvalues for a given regime other than the ones at zero are assumed to be distinct [12]. In [13], we obtain a computationally stable numerical algorithm for the steady-state solution of multiregime FMFQs based on Schur decompositions without having any assumptions on eigenvalue multiplicity. However, there are two scenarios that were not considered in [12] or [13] and we find them crucial in the modeling of the optical buffer of interest:

- For a given state $m$, if the drift is increasing in two neighboring regimes $i$ and $i+1$, i.e., $r_{m}^{(i)}$ $>0, r_{m}^{(i+1)}>0$, then we allow in this paper the drift at the boundary to be zero, i.e., $\widetilde{r}_{m}^{(i)}=0$.

- We allow the infinitesimal generator for a given regime $k$, i.e., $Q^{(k)}$, to be a transient generator.

In this paper, we reduce the analysis of the optical buffering system of interest to the solution of an FMFQ whose parameterization is presented in the next section. We provide a numerical solution for the FMFQ in the Appendix with these two scenarios also taken into account.

\section{StOchastic ANALysis OF OptiCAl BUfFERS}

Recall from Section I that the optical packet arrival process is a MAP characterized with a pair of two $d$ $\times d$ matrices $\left(D_{0}, D_{1}\right)$ and the packet lengths are PHtype distributed, which is characterized with the pair $(v, S)$, where $v$ is a $1 \times s$ vector, $S$ is a nonsingular $s$ $\times s$ matrix, and $S^{0}=-S e$.

In Fig. 1, a sample path for the horizon process $H(t)$ for the asynchronous optical buffer described in Section $\mathrm{I}$ is given for a particular example of $K=D=2$. Note that this sample path consists of continuous decreases with fixed drifts and upward jumps in between. An upward jump appears in the sample path whenever an optical packet is admitted to the optical buffer. The goal of this article is to model the optical buffer with FMFQs, but upward jumps cannot be directly modeled by FMFQs. For this purpose, we introduce a transformed process $H_{T}\left(t^{\prime}\right)$ in which an individual jump for $H(t)$ at time $t$ to its new value $H\left(t^{+}\right)$ $=D[H(t) / D]+B$ in Eq. (1) is replaced with the sequence of three curves described in Table I. The transformed
TABLE I

Three-Step Procedure for Obtaining the Transformed PROCESS $H_{T}\left(T^{\prime}\right)$

1. If $H_{T}\left(t^{\prime}\right)=0$, then go to step 3 ; otherwise $H_{T}\left(t^{\prime}\right)$ increases steadily with unity drift until it hits the particular value $D\lceil H(t) / D\rceil$.

2. $H_{T}\left(t^{\prime}\right)$ sticks to this particular value for an exponentially distributed amount of time.

3. $H_{T}\left(t^{\prime}\right)$ increases steadily with unity drift until it reaches $D[H(t) / D\rceil+B$.

process $H_{T}\left(t^{\prime}\right)$ for the same example is given in Fig. 3 assuming that Step 2 of the above procedure is to last one time unit for all admitted packets. The solid lines (dotted lines) are for the epochs for which $H_{T}\left(t^{\prime}\right)$ is increasing or staying constant (decreasing). We note that if we only concentrate on the epochs during which $H_{T}\left(t^{\prime}\right)$ is decreasing, then we obtain back the original sample path of $H(t)$. Equivalently, if one can find the steady-state distribution of the process $H_{T}\left(t^{\prime}\right)$, then one can recover that of the process $H(t)$, which is the scope of the current article. The approach we pursue in this paper is to show that the process $H_{T}\left(t^{\prime}\right)$ can be modeled as an FMFQ and solve this system to obtain the steady-state distribution for the transformed process, which in turn gives us the distribution of the unfinished work $H(t)$ from which all performance measures can be obtained, including the packet loss probability, mean delay, etc. For this purpose, we inherit the notation used in Section II and introduce $M=d(s+2)$ states in the background process. For the degenerate case, we define $K+1$ regimes and set boundary points as $T^{(k)}=k D$ for $0 \leqslant k \leqslant K$ and $T^{(K+1)}$ $=\infty$. One could choose $T^{(k)}=D_{k}$ for the nondegenerate case. The drift parameters of the corresponding FMFQ that characterizes the transformed process $H_{T}$ are

$$
\begin{aligned}
R^{(K+1)} & =\operatorname{diag}\left(-I_{d},-I_{d}, I_{s d}\right), \\
R^{(k)} & =\operatorname{diag}\left(-I_{d}, I_{d}, I_{s d}\right), \quad 1 \leqslant k \leqslant K,
\end{aligned}
$$

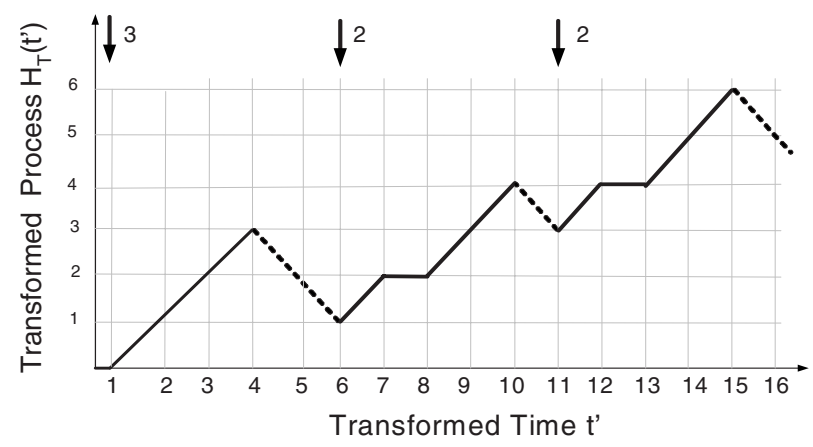

Fig. 3. Evolution of the transformed process $H_{T}\left(t^{\prime}\right)$ for a singlewavelength degenerate optical buffer with $D=K=2$. 


$$
\begin{aligned}
& \widetilde{R}^{(0)}=\operatorname{diag}\left(0_{d}, I_{d}, I_{s d}\right), \\
& \widetilde{R}^{(k)}=\operatorname{diag}\left(-I_{d}, 0_{d}, I_{s d}\right), \quad 1 \leqslant k \leqslant K,
\end{aligned}
$$

where $I_{i}$ and $0_{i}$ denote the identity and the zero matrix of size $i \times i$, respectively, and diag denotes a blockdiagonal matrix obtained through the ordered concatenation of its $\operatorname{arguments} ; \operatorname{diag}(X, Y)$ denotes a matrix $\left[\begin{array}{ll}X & 0 \\ 0 & Y\end{array}\right]$. We now provide the per-regime infinitesimal generators $Q^{(k)}, 1 \leqslant k \leqslant K+1$ :

$$
\begin{aligned}
Q^{(k)} & =\left[\begin{array}{ccc}
D_{0} & D_{1} & 0 \\
0 & 0 & 0 \\
\widetilde{S}^{0} & 0 & \widetilde{S}
\end{array}\right], \quad k \leqslant K, \\
Q^{(K+1)} & =\left[\begin{array}{ccc}
\widetilde{D} & 0 & 0 \\
0 & 0 & 0 \\
\widetilde{S}^{0} & 0 & \widetilde{S}
\end{array}\right],
\end{aligned}
$$

where $\widetilde{S}^{0}=I_{d} \otimes S^{0}, \widetilde{S}=I_{d} \otimes S$, and $\widetilde{D}=D_{0}+D_{1}$. Now, we provide the infinitesimal generators when $C(t)$ is at a boundary point, i.e., $C(t)=T^{(k)}, 0 \leqslant k \leqslant K$ as follows:

$$
\begin{aligned}
\widetilde{Q}^{(k)} & =\left[\begin{array}{ccc}
D_{0} & D_{1} & 0 \\
0 & -I_{d} & \widetilde{V} \\
\widetilde{S}^{0} & 0 & \widetilde{S}
\end{array}\right], \quad k>0, \\
\widetilde{Q}^{(0)} & =\left[\begin{array}{ccc}
D_{0} & 0 & \widetilde{D}_{1} \\
0 & 0 & 0 \\
\widetilde{S}^{0} & 0 & \widetilde{S}
\end{array}\right],
\end{aligned}
$$

where $\widetilde{V}=I_{d} \otimes v$ and $\widetilde{D}_{1}=D_{1} \otimes v$.

The expressions (9)-(16) completely characterize an FMFQ that models the evolution of the transformed process $H_{T}$. To see this, note that we have three sets of states, namely, $\mathbf{A}, \mathbf{B}$, and $\mathbf{C}$. The set $\mathbf{A}$ $=\left\{\mathbf{A}_{1}, \mathbf{A}_{2}, \ldots, \mathbf{A}_{d}\right\}$ comprises the first $d$ states of the FMFQ, i.e., $\{1,2, \ldots, d\}$. On the other hand, the set $\mathbf{B}=\left\{\mathbf{B}_{1}, \mathbf{B}_{2}, \ldots, \mathbf{B}_{d}\right\}$ comprises the set of states $\{d$ $+1, d+2, \ldots, 2 d\}$ of the FMFQ. Finally, the set $\mathbf{C}$ $=\left\{\mathbf{C}_{11}, \mathbf{C}_{12}, \ldots, \mathbf{C}_{1 s}, \mathbf{C}_{21}, \ldots, \mathbf{C}_{d s}\right\}$ consists of the states $\{2 d+1,2 d+2, \ldots, 2 d+s, 2 d+s+1, \ldots, 2 d+d s\}$ of the FMFQ. When the FMFQ is in state $\mathbf{A}_{i}$, then $H_{T}$ is decreasing at a unity rate and the arrival MAP is in state $i$. On the other hand, when the FMFQ is in state $\mathbf{B}_{i}$, then $H_{T}$ is increasing at a unity rate, the next state of the arrival process after the last arrival is $i$, and we note that this increase corresponds to the increase written in Step 1 of the procedure in Table I. Finally, when the FMFQ is visiting state $\mathbf{C}_{i j}$, then $H_{T}$ is increasing with the type described in Step 3 of Table $\mathrm{I}$, the service $\mathrm{PH}$-type process describing packet lengths is in state $j$, and the next state of the arrival process after the last arrival is $i$.

Assume we are in regime $k, k \leqslant K$, a packet arrival has just occurred, and the next state of the arrival process is $i$. Then the packet gets accepted to the optical buffer and the FMFQ process transitions to state $\mathbf{B}_{i}$. Depending on the procedure described in Step 1 of Table I, if $H_{T}$ is greater than zero it should increase at a unity rate until it hits the first boundary point without changing state as dictated in the second block rows of Eqs. (10) and (13). The process $H_{T}$ then stays for an exponentially distributed time with mean 1 (governed by Step 2 of Table I) at this boundary point eventually escaping to state $\mathbf{C}_{i j}$ with probability $v_{j}$. This behavior is indicated in the second block rows of Eqs. (12) and (15). According to Step 3 of Table I and as long as the FMFQ is in state $\mathbf{C}_{i j}$ for some $j, H_{T}$ should continue to increase at a unity rate until the corresponding PH-type distribution reaches the absorbing state, which occurs with rate $S^{0}$, after which we transition to $\mathbf{A}_{i}$. This behavior is captured by the last block rows of Eqs. (9), (10), and (13)-(15). This increase in the queue length should not be paused if any boundary points are crossed when the queue is increasing; this behavior is captured by a drift choice of unity for all $\mathbf{C}$ states as given in Eq. (12). When in state $\mathbf{A}_{i}$, the queue length starts to decrease at a unity rate and the arrival process starts to evolve according to the MAP parameters $D_{0}$ and $D_{1}$. If an arrival occurs when in regime $K+1$, this packet does not get accepted and $H_{T}$ continues to decrease as the MAP continues to evolve. This behavior is captured in the first block rows of Eqs. (9) and (14). If a new packet arrival occurs when the FMFQ is in regime $k, k \leqslant K$, and the next state of the MAP process is $i$, then the FMFQ transitions to state $\mathbf{B}_{i}$; we capture this behavior by the first block row choice of Eq. (13). On the other hand, if an arrival occurs at boundary point $T^{0}=0$ and when the next state of the MAP process is $i$, then the arriving packet is forwarded over the direct link bypassing the Steps 1 and 2 of the procedure of Table I and therefore the corresponding FMFQ transitions to state $\mathbf{C}_{i j}$ with probability $v_{j}$. We capture this behavior with the choice of the first block row of Eq. (16). There are also some situations that need to be avoided. For example, the FMFQ cannot be at a $\mathbf{B}$ state at regime $K+1$ during normal operation, so precautions should be taken to escape from that situation, which leads us to the choices of the second block rows of Eqs. (9) and (14). Similarly, one cannot be at a $\mathbf{B}$ state at threshold $T^{0}=0$, so policies to escape from this situation need to be in place. The choices of the second block rows of Eqs. (11) and (16) stem from this observation. At this point, we complete the characterization of the FMFQ for the process $H_{T}$ while noting that there might be other characterizations for the same FMFQ. 
We provide a computationally stable and efficient algorithm for this FMFQ in the Appendix. Using this method, one can find the steady-state joint CDF $F_{m}(y)=\lim _{t \rightarrow \infty} P\left(H_{T}(t) \leqslant y, M(t)=m\right), 1 \leqslant m \leqslant M=d(s+2)$ for the transformed process $H_{T}$. By conditioning on the $\mathbf{A}$ states during which the queue is decreasing, we can find the steady-state $\mathrm{CDF} G_{j}(y)=\lim _{t \rightarrow \infty} P(H(t)$ $\leqslant y, J(t)=j)(1 \leqslant j \leqslant d)$ for the original process $H$ as follows:

$$
G_{j}(y)=\frac{F_{j}(y)}{\sum_{j=1}^{d} F_{j}(\infty)},
$$

where $J(t)$ is the background process for the arrival MAP. Let $G(y)$ denote the steady-state joint CDF vector $G(y)=\left[G_{1}(y), \ldots, G_{d}(y)\right]$. Let $\widetilde{G}(y)$ denote the steady-state joint complementary CDF vector $\widetilde{G}(y)$ $=\left[\widetilde{G}_{1}(y), \ldots, \widetilde{G}_{d}(y)\right]$, where $\widetilde{G}_{m}(y)=\lim _{t \rightarrow \infty} P(H(t)>y$, $J(t)=m), 1 \leqslant j \leqslant d$, and therefore

$$
\widetilde{G}_{j}(y)=G_{j}(\infty)-G_{j}(y), \quad 1 \leqslant j \leqslant d .
$$

Packet losses occur when $H(t)$ exceeds $T^{(K)}=K D$. Therefore, the packet blocking probability $P_{b}$ is given by

$$
P_{b}=\frac{\widetilde{G}(K D) D_{1} e}{\pi D_{1} e} .
$$

The expression for the expected delay of a packet that is admitted into the optical buffer is then given by

$$
E[\text { Delay }]=\frac{\sum_{k=1}^{K} k D(\hat{G}(k D)-\hat{G}((k-1) D))}{1-P_{b}},
$$

where

$$
\hat{G}(y)=\frac{G(y) D_{1} e}{\pi D_{1} e}
$$

denotes the steady-state probability that an arbitrary arriving packet finds less than $y$ amount of unfinished work in the system. The steady-state probability that the unfinished work in the system is less than $y$ at an arbitrary epoch is obviously different for MAP arrivals and it is equal to $G(y) e$.

\section{NUMERICAL RESULTS}

In this section, we will provide several numerical examples to verify and validate the approach proposed in this paper.

\section{A. Example 1}

As the first example, we study a degenerate optical buffer under Poisson input with intensity $\lambda$ and exponential packet lengths, which is the same problem studied in [6]. For the sake of convenience, the mean packet lengths are normalized to unity and $\lambda$ is first set to 0.8 . For three different values of $K$, we find the packet blocking probabilities for different values of the granularity parameter $D$. The results are given in Fig. 4. The results are in accordance with the simulation results given in [6] and we show that we can obtain very accurate results even for very large $K \mathrm{dem}$ onstrating the numerical stability of the proposed approach.

For a given buffer size $K$ and Poisson packet traffic, there appears to be an optimal value of $D$, denoted by $D^{*}$, under which the packet blocking probability $P_{b}$ is minimized. To explain this and referring to [6], in the first situation of small $D$, the overall buffering capacity will also be low due to fixed $K$, and optical buffering would not be beneficial in this regime. On the other hand, in the situation of large $D$, the amount of work introduced by an individual packet to the system in addition to its length (each jump in Fig. 1 amounts to the work introduced by each admitted packet) would also get larger without bounds, which also leads to reduced performance in terms of packet loss. Between these two situations, a minimum for packet loss is realized with a suitable choice of the granularity parameter, i.e., $D^{*}$. As in $[6,8]$, researchers attempt to try to estimate $D^{*}$ by means of traffic modeling, analysis, and simulations to help provisioning optical buffers. Table II provides the optimum granularity parameter $D^{*}$ obtained through analysis for various values of $K$ and for two different values of $\lambda$. When $\lambda$ is fixed, with increasing $K, D^{*}$ appears to decrease, whereas the largest FDL length $K D^{*}$ increases. We explain this as follows. The optimal use of resources is attained when we take advantage of the increase in $K$ both by increasing the largest FDL length and hence

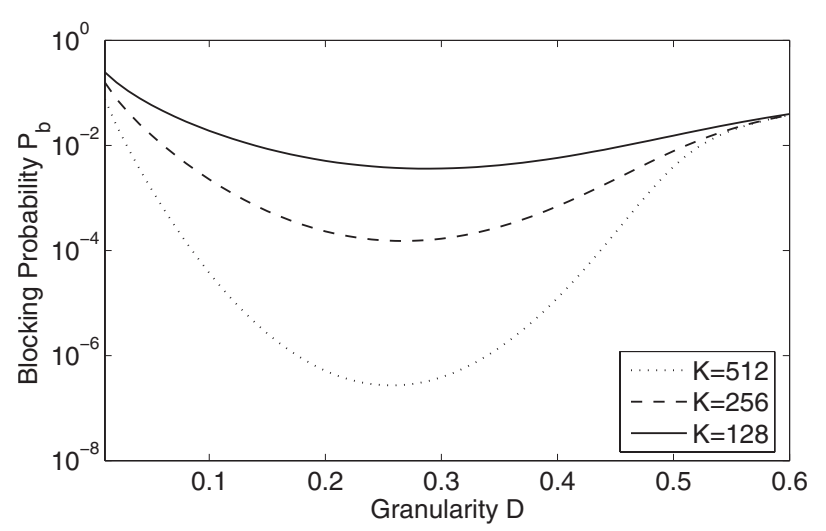

Fig. 4. Packet blocking probability $P_{b}$ as a function of the granularity parameter $D$ for varying values of the buffer size $K$. 
TABLE II

The Optimum Granularity Parameter $D^{*}$ For VARIOUS VAlues of $K$ AND FOR Two VAlues of $\lambda$

\begin{tabular}{rrrrrc}
\hline$\lambda$ & $K$ & $D^{*}$ & $\lambda$ & $K$ & $D^{*}$ \\
\hline 0.8 & 32 & 0.395 & 0.6 & 16 & 0.773 \\
& 64 & 0.329 & & 32 & 0.704 \\
& 128 & 0.288 & & 64 & 0.669 \\
& 256 & 0.267 & & 96 & 0.659 \\
& 512 & 0.257 & & 128 & 0.654 \\
\hline
\end{tabular}

increasing the buffering capacity (situation 1 described above) and by also reducing the granularity parameter (situation 2 described above). When $K$ is fixed, $D^{*}$ appears to increase with decreasing $\lambda$. When $\lambda$ is relatively low, the excess load generated by larger $D$ seems to be tolerable in terms of system performance. More conclusive studies are carried out for optimal choice of granularity in Examples 4 and 5.

\section{B. Example 2}

In this example, we find the steady-state $\operatorname{PDF} \hat{g}(y)$ $=\mathrm{d} \hat{G}(y) / \mathrm{d} y$ of the unfinished work that an arriving optical packet finds as a function of $y$ as a function of the unfinished work $y$. Note that, all performance measures of interest can be derived from this PDF. We consider a degenerate optical buffer comprising $K=2$ FDLs with $D=1$. Our goal in this example is to show that the distribution of the unfinished work in the system can exactly be calculated by the proposed method. The packet lengths are assumed to be of PH-type distribution that is characterized with the pair $(v, S)$ :

$$
S=\left[\begin{array}{cc}
-20 & 10 \\
10 & -10
\end{array}\right], \quad v=\left[\begin{array}{ll}
0.5 & 0.5
\end{array}\right],
$$

for which the mean packet length is 0.25 . In this example, packet lengths are exponentially distributed with mean 0.2618 with probability 0.9472 , and they are exponentially distributed with mean 0.0038 with probability 0.0528 . Hence, this particular distribution can be viewed to model two different modes for packet lengths, i.e., short and long packets. Note that timerbased burstifiers generate short bursts in low loads and long bursts in high loads. Moreover, for this particular example, the optical packet arrival process is assumed to be a MAP characterized with the pair $\left(D_{0}, D_{1}\right)$ :

$$
D_{0}=\frac{1}{a}\left[\begin{array}{ccc}
-5 & 1 & 1 \\
1 & -5 & 1 \\
1 & 1 & -5
\end{array}\right], \quad D_{1}=\frac{1}{a}\left[\begin{array}{ccc}
1 & 2 & 0 \\
1 & 1 & 1 \\
1 & 1 & 1
\end{array}\right],
$$

where $a$ is a varying parameter. The mean packet arrival intensity is written as $3 / a$ and therefore the load on the system is $3 /(4 a)$. Figure 5 illustrates the PDF

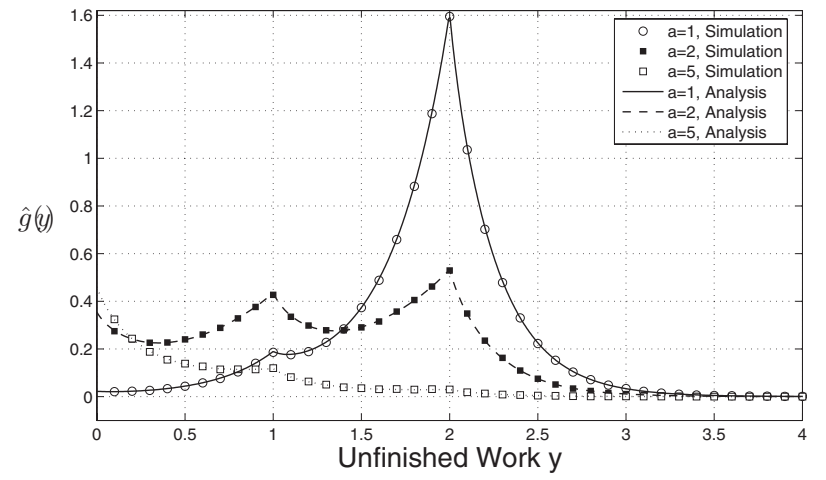

Fig. 5. PDF, $\hat{g}(y)$, of the unfinished work that an arriving packet finds for Example 2 for varying values of $a$.

$\hat{g}(y), y>0$ for varying values of $a$, and we show that the analytical results are in perfect accordance with the simulation results. Note that probability masses at the origin are not depicted in this figure. When the parameter $a$ increases, the load on the system decreases and the need for using FDLs is reduced. For $a=1$, which amounts to a relatively high load of $3 / 4$, there is a significant peak at $y=2$, which means that an arriving packet will (most probably) either get blocked or use the larger FDL of length 2 . When the load decreases, for example, when $a=2$, both FDLs appear to be equally usable; note the two peaks at $y=1$ and $y=2$. In case of relatively low loads, for example, when $a=5$, the unfinished work upon an arrival is zero most of the time and most of the packets get admitted and few of them get delayed. This example demonstrates that we not only find the blocking probabilities but also the entire PDF by means of the proposed approach for general PH-distributed packet lengths and MAP-type packet arrivals.

In the next examples, we would like to draw conclusions on how packet length distributions and packet interarrival time distributions affect the performance of the system and see whether we can build provisioning guidelines for optical buffers.

\section{Example 3}

In this example, we study the affect of the squared coefficient of variation (SCV) of packet lengths on blocking performance when packet arrivals are Poisson. The SCV of a random variable is the variance divided by the squared mean of that random variable and is indicative of its variability. For exponentially distributed packet lengths, we have $\mathrm{SCV}=1$. On the other hand, the Erlang- $k$ distribution with $k$ phases with $\mathrm{SCV}=1 / k$ can be used to model scenarios when $\mathrm{SCV}<1$. The scenario SCV $>1$ can be modeled by using an appropriate two-phase hyper-exponential distribution (denoted by $\mathrm{H}_{2}$ ) with balanced means ([24], pp. 58-59). Both the Erlang- $k$ and hyper-exponential distributions are of PH-type and can be addressed in 
the framework of the current article. We fix $K=20$ and we plot the packet blocking probability as a function of the granularity $D$ for different values of SCV under three different loads $\rho=0.8,0.6,0.4$ in Fig. 6 . The results are also in line with the results obtained by [8]. We have the following observations:

- As the packet length SCV increases, then the packet loss probabilities tend to increase for a wide range of the granularity parameter $D$, which leads us to believe that packetization policies attempting to minimize the SCV are needed to address this performance impact. This relationship appears to be reversed for very large values of $D$, but this particular regime of large granularities needs to be avoided due to high blocking probabilities and delays arising in this regime.

- The optimal granularity parameter that leads to minimal blocking probabilities, say $D^{*}$, increases with increased load and increased packet length SCV.

\section{Example 4}

In this example, we focus on Poisson packet arrivals and exponential packet lengths and we then study the choice of the optimal granularity parameter $D^{*}$ as a function of the buffer size $K$ that maximizes the throughput of the system under a desired blocking probability of $P_{b}$. For this purpose, for given $K$ and $D$, we gradually change the Poisson arrival intensity such that the desired blocking probability $P_{b}$ is achieved and we denote this particular intensity by $\lambda\left(D, K, P_{b}\right)$. The throughput of this system is then given by $\lambda\left(D, K, P_{b}\right)\left(1-P_{b}\right)$. The optimal granularity $D^{*}$ is the value of $D$ that maximizes $\lambda\left(D, K, P_{b}\right)(1$ $-P_{b}$ ) for a given $K$ and desired blocking probability $P_{b}$. We fix $P_{b}=10^{-4}$ and we plot $\lambda\left(D, K, P_{b}\right)\left(1-P_{b}\right)$ for exponential packet lengths in Fig. 7(a) as a function of $D$ and for various values of $K$. Similar to Example 1, $D^{*}$ decreases with increasing $K$. For this problem, we also seek a provisioning guideline for properly choosing the granularity parameter $D$. For this purpose, we also plot $D^{*} \sqrt{K}$ as a function of the buffer size $K$ in Fig. 7(b) for various values of $P_{b}$ and packet length SCV. Our results show that the function $D^{*} \sqrt{K}$ has a relatively narrow dynamic range for varying $K$. Then the optimal granularity parameter $D^{*}$ approximately behaves as

$$
D^{*} \propto K^{-1 / 2}
$$

for the four scenarios we studied. The proportionality constant in the above relation appears to decrease with decreasing $P_{b}$ and with increasing packet length SCV. To give an example, let us fix $P_{b}=10^{-4}$ and $\mathrm{SCV}=1$. From Fig. 7(b), $D^{*}$ behaves approximately as $5 / \sqrt{K}$ for relatively large values of $K$, and when $K$
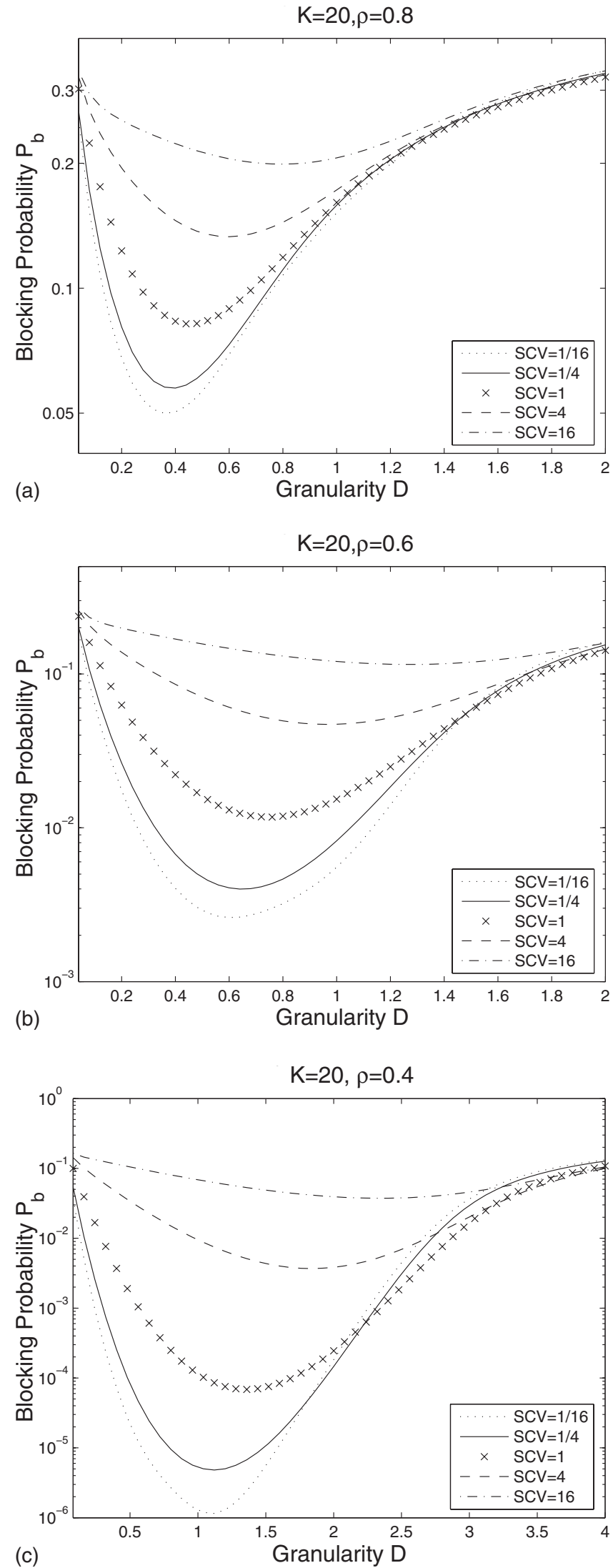

Fig. 6. Blocking probability $P_{b}$ for $K=20$ as a function of the granularity parameter $D$ for autocorrelation with different values of packet length SCV (a) $\rho=0.8$, (b) $\rho=0.6$, (c) $\rho=0.4$. 


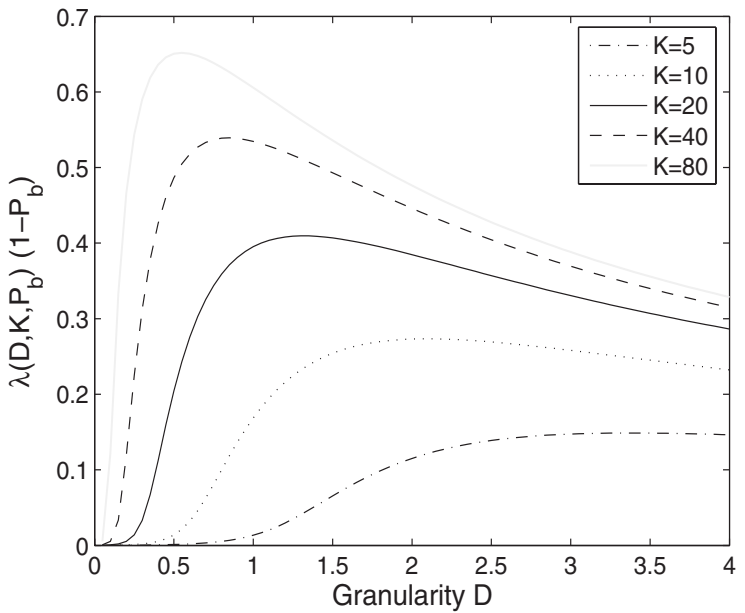

(a)

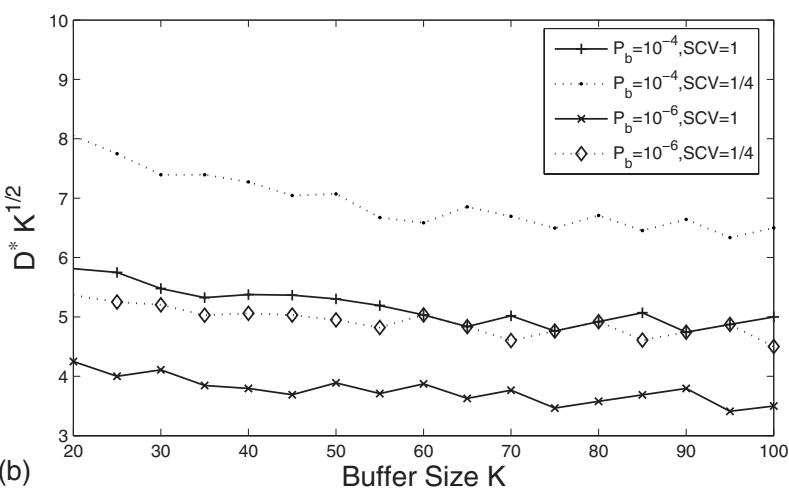

Fig. 7. The plot of (a) $\lambda\left(D, K, P_{b}\right)\left(1-P_{b}\right)$ as a function of $D$ for various $K$ for a desired blocking probability of $P_{b}=10^{-4}$. (b) The quantity $D^{*} \sqrt{K}$ as a function of the buffer size $K$ for two different values of $P_{b}$ and packet length SCV.

$=80$, the estimate of the granularity parameter equals $5 / \sqrt{80}=0.56$, which is very close to the actual optimal value as depicted in Fig. 7(a).

\section{E. Example 5}

This example is similar to the previous example except that we have a constraint on the total fiber size $L$ to be used for FDLs in a way that $L=D K(K+1) / 2$. Again we focus on Poisson packet arrivals and exponential packet lengths, and we then study the choice of the optimal granularity parameter $D^{*}$, this time as a function of the fiber size $L$ that maximizes the throughput of the system for a desired blocking probability $P_{b}$. For this purpose, for given $L$ and $K$, we vary the Poisson arrival intensity until a desired blocking probability $P_{b}$ is achieved and we denote this particular intensity by $\lambda^{0}\left(L, K, P_{b}\right)$. The throughput of this system is then given by $\lambda^{0}\left(L, K, P_{b}\right)\left(1-P_{b}\right)$. The optimal buffer size $K^{*}$ is the value of $K$ that maximizes $\lambda^{0}\left(L, K, P_{b}\right)\left(1-P_{b}\right)$ for a given $L$ and desired blocking probability $P_{b}$. We fix $P_{b}=10^{-4}$ and we plot $\lambda^{0}\left(L, K, P_{b}\right)\left(1-P_{b}\right)$ for exponential packet lengths in Fig. 8(a) as a function of $K$ and for various values of $L$.
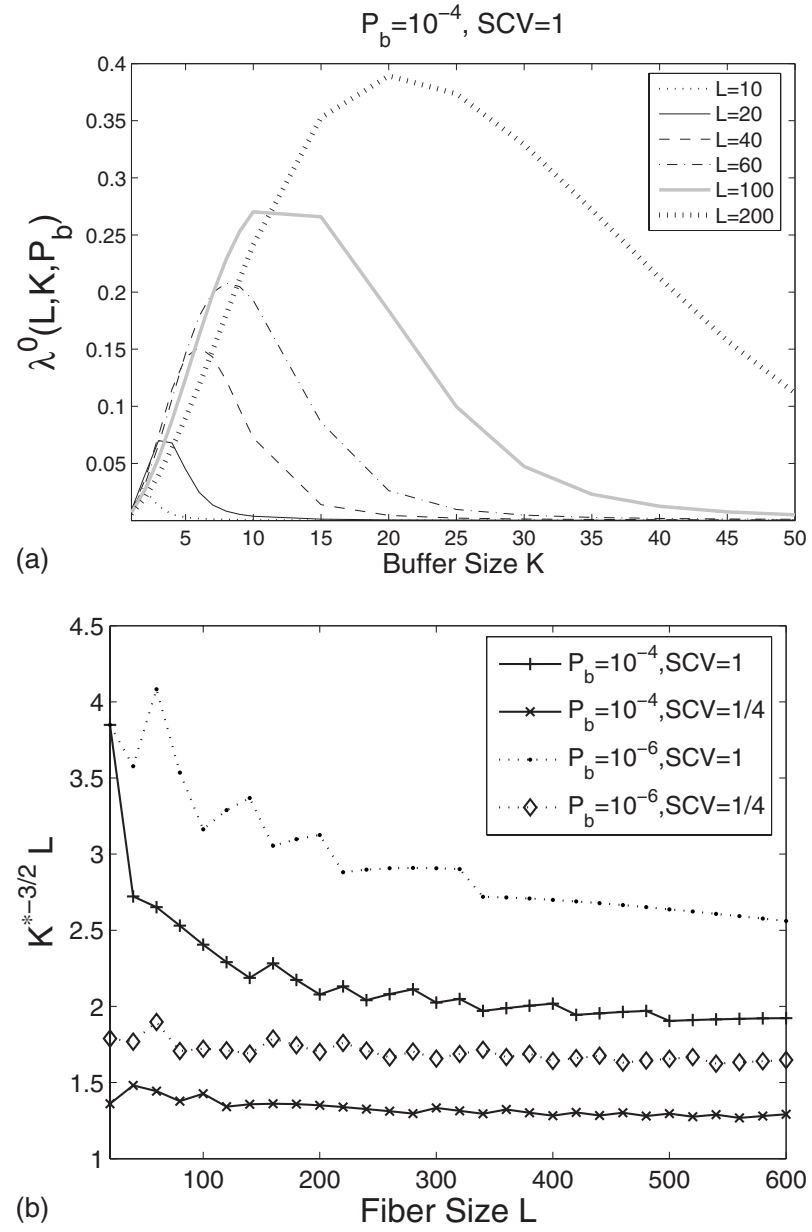

Fig. 8. The plot of (a) $\lambda^{0}\left(L, K, P_{b}\right)\left(1-P_{b}\right)$ as a function of $K$ for various values of the total fiber size $L$ when $P_{b}=10^{-4}$ and $\mathrm{SVC}=1$ and (b) $\left(K^{*}\right)^{-3 / 2} L$ as a function of the total fiber size $L$ for two different values of $P_{b}$ and packet length SCV.

It is clear that $K^{*}$ increases with increasing fiber size $L$. It is interesting to note that there are cases when the achievable throughput drops as a result of an increase in the total fiber size $L$ if the buffer size stays $K$ fixed. This observation shows that the buffer size selection is very critical. One of the goals of this work is to find a provisioning guideline for buffer size selection. For this purpose, we also plot $\left(K^{*}\right)^{-3 / 2} L$ as a function of the fiber size $L$ in Fig. 8(b) for various values of $P_{b}$ and packet length SCV. Our results show that the function $\left(K^{*}\right)^{-3 / 2} L$ has a relatively narrow dynamic range for varying $L$ and the optimal buffer size parameter $K^{*}$ behaves as

$$
K^{*} \propto L^{2 / 3}
$$

for the scenarios we studied. The proportionality constant in the above relation appears to decrease with decreasing $P_{b}$ and with increasing SCV. To give an example, let us fix $P_{b}=10^{-4}$ and SCV=1. From Fig. 8(b), $K^{*}$ behaves approximately as $2^{-2 / 3} L^{2 / 3}$ for relatively large values of $L$, and when $L=200$, the estimate of the optimal buffer size becomes $2^{-2 / 3} 200^{2 / 3}=21.54$, 
which is very close to the actual optimal value as depicted in Fig. 8(a).

\section{F. Example 6}

In this example, we study the effect of autocorrelation on packet blocking probability. The interarrival distribution is hyper-exponential with balanced means, but the autocovariance function $C(k)$ of the interarrival times is of the form $A \sigma^{k}$ for some constant $A$. Such an arrival process can be obtained by a twophase MAP using the procedure of [25]. The larger the parameter $\sigma$, the more dominant the autocorrelation is. We assume exponential packet lengths again with unity mean. We fix $\rho=0.25$ and plot the blocking probability $P_{b}$ against the granularity $D$ for various values of $\sigma$ in Fig. 9. It is clear that autocorrelation adversely affects the blocking rates, but the optimal granularity parameter also decreases with increased $\sigma$. We leave a more detailed discussion of autocorrelated traffic modeling in optical packet switched networks for future research.

\section{CONCLUSION}

We have provided an exact analytical procedure to solve single-wavelength optical buffers with MAP traffic input and phase-type distributed packet lengths. We provided numerical examples to give insight for the operation of the optical buffer, and furthermore we provided provisioning guidelines for optical buffers for improved performance.

\section{APPENDIX}

Here, we provide a solution methodology for the FMFQ described through Eqs. (9)-(16). Using the results obtained in [13], we can list the boundary condi-

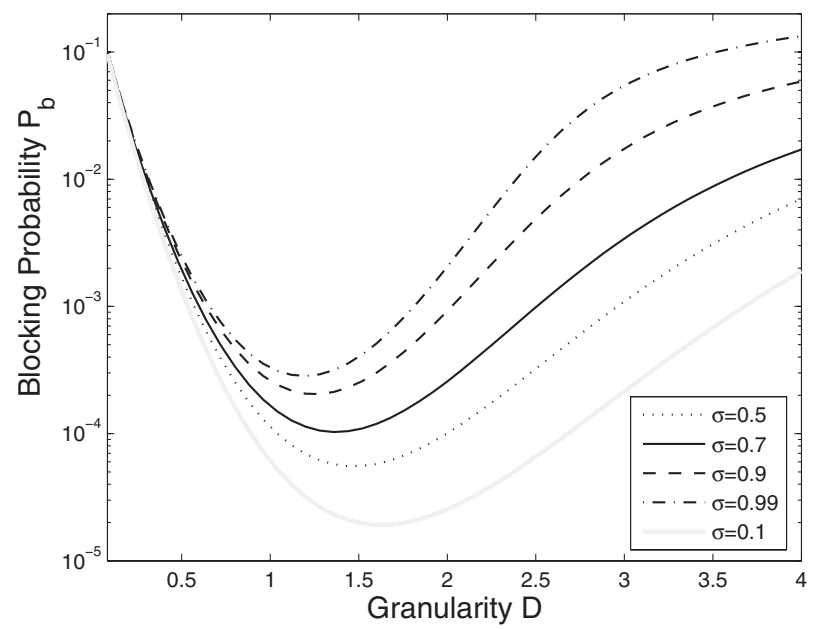

Fig. 9. Blocking probability $P_{b}$ against $D$ for various values of $\sigma$ when $\lambda=0.25$. tions for the FMFQ of interest in terms of the functions $f^{(k)}, 1 \leqslant k \leqslant K$, and $c^{(k)}$, as follows:

$$
\begin{gathered}
f^{(k+1)}\left(T^{(k)}+\right) R^{(k+1)}-f^{(k)}\left(T^{(k)}-\right) R^{(k)}=c^{(k)} \widetilde{\mathbf{Q}}^{(k)}, \\
1 \leqslant k \leqslant K \\
f_{m}^{(k+1)}\left(T^{(k)}+\right)=0, \quad m \in \mathbf{B}, \quad 1 \leqslant k \leqslant K, \\
c_{m}^{(k)}=0, \quad m \in \mathbf{A} \cup \mathbf{C}, \quad 1 \leqslant k \leqslant K, \\
f^{(1)}(0+) R^{(1)}=c^{(0)} \widetilde{Q}^{(0)}, \\
c_{m}^{(0)}=0, \quad m \in \mathbf{C} .
\end{gathered}
$$

We note that in [13], we did not have a set like $\mathbf{B}$ where the drifts for that state in two subsequent regimes have the same sign but are zero at the interim boundary point. Therefore, corresponding boundary conditions were not given in that study. Following the same lines of the proof in the appendix of [13], one can easily obtain the additional boundary condition (A.2), the proof of which is omitted in the current article. Again, based on [13] and Section III of the current article, the steady-state joint PDF vector of the FMFQ of interest, $f^{(k)}(y)$, satisfies the following differential equation for $1 \leqslant k \leqslant K+1$ :

$$
\frac{\mathrm{d}}{\mathrm{d} y} f^{(k)}(y)=f^{(k)}(y) \underbrace{Q^{(k)}\left(R^{(k)}\right)^{-1}}_{A^{(k)}}, \quad T^{(k-1)}<y<T^{(k)},
$$

where

$$
\begin{aligned}
A^{(k)}=A & =\left[\begin{array}{ccc}
-D_{0} & D_{1} & 0 \\
0 & 0 & 0 \\
-\widetilde{S}^{0} & 0 & \widetilde{S}
\end{array}\right], \quad 1 \leqslant k \leqslant K \\
& =\widetilde{A}=\left[\begin{array}{ccc}
-\widetilde{D} & 0 & 0 \\
0 & 0 & 0 \\
-\widetilde{S}^{0} & 0 & \widetilde{S}
\end{array}\right], \quad k=K+1 .
\end{aligned}
$$

Note that $D_{0}$ and $\widetilde{S}$ are substochastic matrices and all their eigenvalues are in the open left half-plane. Therefore, the matrix $-D_{0}$ has all its eigenvalues in the right half-plane. On the other hand, $D$ is a stochastic matrix and all its eigenvalues are in the open left half-plane except for one that resides at the origin. Now, let

$$
S_{1}=\left[\begin{array}{ll}
-\widetilde{S}^{0} & 0
\end{array}\right]
$$




$$
\begin{aligned}
& S_{2}=\left[\begin{array}{cc}
-D_{0} & D_{1} \\
0 & 0
\end{array}\right], \\
& \widetilde{S}_{2}=\left[\begin{array}{cc}
-\widetilde{D} & 0 \\
0 & 0
\end{array}\right],
\end{aligned}
$$

be $s d \times 2 d, 2 d \times 2 d$, and $2 d \times 2 d$ matrices, respectively. Let $X_{1}$ be the solution of the Sylvester matrix equation (see [26], Ch. 7),

$$
-\widetilde{S} X_{1}+X_{1} S_{2}+S_{1}=0,
$$

and $X_{2}=-D_{0}^{-1} D_{1}$. The existence of a unique solution $X_{1}$ stems from the spectrum disjointness of $D_{0}$ and $-\widetilde{S}$. Also let $\widetilde{X}_{1}$ be the solution of the Sylvester matrix equation

$$
-\widetilde{S} \widetilde{X}_{1}+\widetilde{X}_{1} \widetilde{S}_{2}+S_{1}=0 .
$$

Let us also suitably partition $X_{1}=\left[X_{11}, X_{12}\right]$ and $\tilde{X}_{1}$ $=\left[\widetilde{X}_{11}, \widetilde{X}_{12}\right]$. With the choices of two matrices

$$
\begin{gathered}
Y=\left[\begin{array}{ccc}
X_{11} & X_{12} & I \\
I & X_{2} & 0 \\
0 & I & 0
\end{array}\right], \\
\tilde{Y}=\left[\begin{array}{ccc}
\tilde{X}_{11} & \tilde{X}_{12} & I \\
I & 0 & 0 \\
0 & I & 0
\end{array}\right]
\end{gathered}
$$

one can show by direct substitution that

$$
\begin{aligned}
& Y A Y^{-1}=\operatorname{diag}\left(\widetilde{S},-D_{0}, 0_{d}\right), \\
& \tilde{Y} \tilde{A} \tilde{Y}^{-1}=\operatorname{diag}\left(\widetilde{S},-\widetilde{D}, 0_{d}\right) .
\end{aligned}
$$

Based on the formulation given in the Appendix of [13], we are now ready to write the steady-state joint $\mathrm{PDF}$ vector as

$$
\begin{gathered}
f^{(k)}(y)=a_{-}^{(k)} e^{\tilde{S}\left(y-T^{(k-1)}\right)} L_{-}+a_{+}^{(k)} e^{D_{0}\left(T^{(k)}-y\right)} L_{+}+a_{0}^{(k)} L_{0}, \\
1 \leqslant k \leqslant K,
\end{gathered}
$$

where $L_{-}, L_{+}$, and $L_{0}$ are the first, second, and third block rows of the matrix $Y$ in Eq. (A.11), respectively, and $a_{-}^{(k)}, a_{+}^{(k)}$, and $a_{0}^{(k)}, 1 \leqslant k \leqslant K$, are row vectors of coefficients of size $s d, d$, and $d$, respectively, and are to be found using the boundary conditions (A.1)-(A.5). Similarly,

$$
f^{(K+1)}(y)=a_{-}^{(K+1)} e^{\tilde{S}\left(y-T^{(K)}\right)} \tilde{L}_{-},
$$

where $\widetilde{L}_{-}$is the first block row of the matrix $\tilde{Y}$ in Eq. (A.12), and $a_{-}^{(K+1)}$ is a row vector of coefficients of size $s d$ that is to be found using the boundary conditions (A.1)-(A.5). We note that the other modes of the ma- trix $\widetilde{A}$ should not be excited since otherwise the solution would grow without bounds or the probabilities would not add up to unity. We have $d(s+2) K+d s$ unknowns from Eqs. (A.15) and (A.16) and $(K+1) d(s$ $+2)$ unknown probability mass accumulation vectors $c^{(k)}, 0 \leqslant k \leqslant K$, that are to be found based on the boundary conditions (A.1)-(A.5). Overall, we have $2(K$ $+1) M-2 d$ unknowns. Let us try to find the number of equations available to use. The boundary condition (A.1) gives $K M$ equations. On the other hand, the boundary conditions (A.2) and (A.3) provide $K M$ more equations. The boundary conditions (A.4) and (A.5) provide $M$ and $d s+s$ equations totalling $2(K+1) M$ $-2 d$ equations. However, one of these equations is redundant (see [13]) and we need a further normalizing equation:

$$
\sum_{m=1}^{M}\left(\sum_{k=1}^{K+1} \int_{T^{(k-1)_{+}}}^{T^{(k)} f_{m}} f_{m}^{(k)}(x) \mathrm{d} x+\sum_{k=0}^{K} c_{m}^{(k)}\right)=1 .
$$

The above equation can further be written in terms of the unknown as

$$
\begin{aligned}
1= & \sum_{k=0}^{K} c^{(k)} e+\sum_{k=1}^{K} a_{0}^{(k)} L_{0} e+\sum_{k=1}^{K} a_{-}^{(k)}\left(e^{\tilde{S} \Delta^{(K)}}-I\right) \widetilde{S}^{-1} L_{-} e \\
& -a_{-}^{(K+1)} \widetilde{S}^{-1} \widetilde{L}_{-} e+\sum_{k=1}^{K} a_{+}^{(k)}\left(e^{D_{0} \Delta^{(K)}}-I\right) D_{0}^{-1} L_{+} e
\end{aligned}
$$

where $\Delta^{(k)}=T^{(k)}-T^{(k-1)}$, and in the nondegenerate case it should reduce to $\Delta^{(k)}=D$. Note that the proposed procedure reduces to the solution of a linear matrix equation of size $K(s+3) d+d(s+2)$ in the most general case, which further reduces to the solution of an equation of size $4 K+3$ when arrivals are Poisson and packet lengths are exponential.

\section{ACKNOWLEDGMENT}

This work was supported in part by the Science and Research Council of Turkey (Tübitak) under project no. EEEAG-106E046 and with the support of the BONE project (Building the Future Optical Network in Europe), a Network of Excellence funded by the European Commission through the 7th ICT-Framework Programme

\section{REFERENCES}

[1] P. Gambini, M. Renaud, C. Guillemot, F. Callegati, I. Andonovic, B. Bostica, D. Chiaroni, G. Corazza, S. L. Danielsen, P. Gravey, P. B. Hansen, M. Henry, C. Janz, A. Kloch, R. Krahenbuhl, C. Raffaelli, M. Schilling, A. Talneau, and L. Zucchelli, "Transparent optical packet switching: network architecture and demonstrators in the KEOPS project," IEEE J. Sel. Areas Commun., vol. 16, pp. 1245-1259, 1998.

[2] C. Qiao and M. Yoo, "Optical burst switching (OBS)—a new paradigm for an optical Internet," J. High Speed Networks (JHSN), vol. 8, no. 1, pp. 69-84, 1999.

[3] R. Barry and P. Humblet, "Models of blocking probability in 
all-optical networks with and without wavelength changers," IEEE J. Sel. Areas Commun., vol. 14, no. 5, pp. 858-867, June 1996.

[4] I. Chlamtac, A. Fumagalli, L. Kazovsky, P. Melman, W. Nelson, P. Poggiolini, M. Cerisola, A. Choudhury, T. Fong, R. Hofmeister, C.-L. Lu, A. Mekkittikul, D. J. M. Sabido IX, C.-J. Suh, and E. Wong, "Cord: contention resolution by delay lines," IEEE J. Sel. Areas Commun., vol. 14, no. 5, pp. 1014-1029, June 1996.

[5] T. Zhang, K. Lu, and J. Jue, "Shared fiber delay line buffers in asynchronous optical packet switches," IEEE J. Sel. Areas Commun., vol. 24, no. 4, pp. 118-127, 2006.

[6] F. Callegati, "Optical buffers for variable length packets," IEEE Commun. Lett., vol. 4, no. 9, pp. 292-294, Sept. 2000.

[7] R. C. Almeida Jr., J. Pelegrini, and H. Waldman, "A generictraffic optical buffer modeling for asynchronous optical switching networks," IEEE Commun. Lett., vol. 9, no. 2, pp. 175-177, Feb. 2005

[8] W. Rogiest, K. Laevens, D. Fiems, and H. Bruneel, "A performance model for an asynchronous optical buffer," Perform. Eval., vol. 62, no. 1-4, pp. 313-330, 2005.

[9] W. Rogiest, J. Lambert, D. Fiems, B. V. Houdt, H. Bruneel, and C. Blondia, "A unified model for synchronous and asynchronous FDL buffers allowing closed-form solution," Perform. Eval., vol. 66, no. 7, pp. 343-355, 2009.

[10] Z. Liang and S. Xiao, "Performance evaluation of singlewavelength fiber delay line buffer with finite waiting places," J. Lightwave Technol., vol. 26, no. 5, pp. 520-527, Mar. 2008.

[11] J. Liu, T. T. Lee, X. Jiang, and S. Horiguchi, "Blocking and delay analysis of single wavelength optical buffer with general packet size distribution," J. Lightwave Technol., vol. 27, no. 8, pp. 955-966, 2009.

[12] M. Mandjes, D. Mitra, and W. Scheinhardt, "Models of network access using feedback fluid queues," Queueing Syst., vol. 44, no. 4, pp. 2989-3002, 2003.

[13] H. E. Kankaya and N. Akar, "Solving multi-regime feedback fluid queues," Stoch. Models, vol. 24, no. 3, pp. 425-450, July 2008.

[14] M. F. Neuts, Structured Stochastic Matrices of M/G/1 Type and Their Applications. New York: Marcel Dekker, 1989.

[15] D. M. Lucantoni, "New results for the single server queue with a batch Markovian arrival process," Stoch. Models, vol. 7, pp. 1-46, 1991.

[16] V. Paxson and S. Floyd, "Wide-area traffic: the failure of Poisson modeling," IEEE/ACM Trans. Netw., vol. 3, pp. 226-244, 1995.

[17] L. Muscariello, M. Mellia, M. Meo, R. L. Cigno, and M. A. Marsan, "An MMPP-based hierarchical model of Internet traffic," in 2004 IEEE Int. Conf. Communications, vol. 4, June 2004, pp. $2143-2147$.

[18] M. F. Neuts, Matrix-Geometric Solutions in Stochastic Models. Baltimore, MD: Johns Hopkins U. Press, 1981.

[19] S. Asmussen, O. Nerman, and M. Olsson, "Fitting phase-type distributions via the EM algorithm," Scand. J. Stat., vol. 23, pp. 419-441, 1996.

[20] D. Anick, D. Mitra, and M. M. Sondhi, "Stochastic theory of a data handling system with multiple sources," Bell Syst. Tech. J., vol. 61, pp. 1871-1894, 1982.

[21] R. Tucker, "Accurate method for analysis of a packet speech multiplexer with limited delay," IEEE Trans. Commun., vol. 36, no. 4, pp. 479-483, 1988.

[22] I. Adan, E. van Doorn, J. Resing, and W. Scheinhardt, "Analysis of a single-server queue interacting with a fluid reservoir," Queueing Syst., vol. 29, pp. 313-336, 1998.

[23] W. Scheinhardt, "Markov modulated and feedback fluid queues," Ph.D. dissertation, University of Twente, Enschede, The Netherlands, 1998.

[24] B. R. Haverkort, Performance of Computer and Communication Systems: A Model-based Approach. Wiley, 1998.

[25] S. Kang and D. Sung, "Two-state MMPP modeling of ATM superposed traffic streams based on the characterization of correlated interarrival times," in IEEE Global Telecommunications Conf., 1995. GLOBECOM '95, vol. 2, Nov. 1995, pp. 14221426.

[26] G. H. Golub and C. F. van Loan, Matrix Computations, 3rd ed. Baltimore, MD: Johns Hopkins U. Press, 1996.

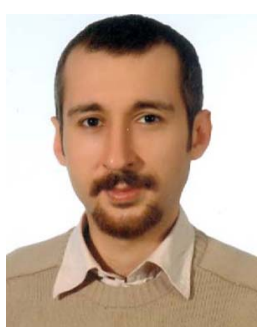

Emre Kankaya received his B.S., M.S., and Ph.D. degrees from the Electrical and Electronics Engineering Department of Bilkent University, Ankara, Turkey, in 2004, 2006, and 2009, respectively. $\mathrm{He}$ is currently an Assistant Professor in the Computer Engineering Department of Zirve University, Gaziantep, Turkey. His research interests include stochastic processes, queueing systems, and performance evaluation of computer networks.

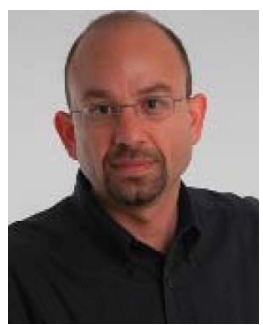

Nail Akar received his B.S. degree from Middle East Technical University, Turkey, in 1987 and his M.S. and Ph.D. degrees from Bilkent University, Turkey, in 1989 and 1994, respectively, all in electrical and electronics engineering. From 1994 to 1996, he was a Visiting Scholar and a Visiting Assistant professor in the Computer Science Telecommunications program at the University of Missouri-Kansas City. In 1996, he joined the Technology Planning and Integration group at the Long Distance Division, Sprint, where he held a Senior Member of Technical Staff position from 1999 to 2000. Since 2000, he has been a faculty member at Bilkent University, currently as an Associate Professor. His current research interests include performance analysis of computer and communication networks, queueing systems, traffic engineering, network control and resource allocation, and optical networking. Dr. Akar actively participates in the European Commission FP7 NoE project BONE. 